\title{
Mudança da tendência da mortalidade por asma em crianças e adolescentes no Rio Grande do Sul: 1970-1998"
}

\author{
José Miguel Chatkin ${ }^{1}$, Jussara Fiterman², Nivaldo Almeida FonseCA ${ }^{3}$, Carlos Cezar FritscheR ${ }^{1}$
}

Introdução: A mortalidade por asma aumentou no Rio Grande do Sul no período 1970-92 em crianças e adultos jovens. Este trabalho visou avaliar a tendência do fenômeno no mesmo grupo etário, agora incluindo o período de 1970-98. Métodos: Foram revisados os 157 certificados de óbitos ocorridos entre 1970 e 1998 em pessoas de cinco a 19 anos de idade nos quais asma foi considerada

a causa básica de morte. As tendências foram testadas usando os modelos log-linear, logístico $(S$ curve) e quadrático. Resultados: A mortalidade por asma variou entre 0,04 e 0,399/100.000. Entre

os modelos testados, o logístico mostrou os melhores valores de acurácia para a série temporal analisada: $\mathrm{r}^{2}=0,59$, percentual de erro médio absoluto (MAPE) $=23,48$, desvio médio absoluto (MAD) $=0,035$ e desvio mínimo quadrático $=0,0021$. Esses resultados significam que possivelmente um platô foi atingido. O modelo quadrático mostrou também bons valores de acurácia, significando que uma possibilidade alternativa seja a de que um decréscimo nos coeficientes esteja iniciando. Neste modelo, o valor máximo calculado foi no $25^{\circ}$ ano (1994). Conclusões: A mortalidade por asma no

Rio Grande do Sul está-se estabilizando, após um período de importante aumento. É possivel, inclusive, que uma tendência ao decréscimo esteja iniciando. (J Pneumol 2001;27(2):89-93)

\section{Change in asthma mortality trends in children and adolescents in Rio Grande do Sul: 1970-1998}

Introduction: During the period from 1970 to 1992, mortality from asthma in children and young adults increased in Rio Grande do Sul. The present study aimed at assessing this phenomenon in patients of the same age group, now extending the time period to 1998.

Methods: The death certificates of 157 patients aged between 5 and 19 years in which asthma was reported to be the cause of death during 1970-80 were reviewed. Testing for trends was conducted using the log-linear, S-curve, and quadratic models. Results: Asthma mortality rate ranged from 0.04 to 0.399/100,000. Among the tested models, $S$ curve trend model showed the best accuracy for the adjusted time series: $r^{2}=0.59$; mean absolute percentage error (MAPE) $=$ 23.48; mean absolute deviation $(M A D)=0.035$; mean square deviation $(M S D)=0.0021$. These results suggest that a plateau has probably been reached. The quadratic model also showed good accuracy values suggesting that a decrease in the coefficients probably started to occur. In this model, the estimated maximal point value was found in the $25^{\text {th }}$ year (1994). Conclusions: Stabilization of asthma death rates is starting to occur in Rio Grande do Sul and it is likely that a decrease will take place.

* Trabalho realizado na Faculdade de Medicina da Pontifícia Universidade Católica do Rio Grande do Sul (PUCRS).

1. Professor Titular de Medicina Interna/Pneumologia.

2. Professora Adjunta de Medicina Interna/Pneumologia.

3. Professor Titular do Departamento de Estatística do Instituto de Matemática.

Endereço para correspondência - Dr. José Miguel Chatkin, Hospital São Lucas da PUCRS, Av. Ipiranga, 6.690, conj. 314 - 90610-000 Porto Alegre, RS. E-mail: jmchatkin@pucrs.br

Recebido para publicação em 27/6/00. Aprovado, após revisão, em 15/9/00.
Descritores - Asma. Mortalidade. Brasil.

Key words - Asthma. Mortality. Brazil.

\section{INTRODUÇÃO}

Apesar dos significativos avanços no entendimento de sua fisiopatogenia e introdução de novas drogas ou esquemas terapêuticos padronizados, a asma é difícil problema de saúde pública na maioria dos países. 
A análise da tendência da mortalidade por asma em diversos países nas últimas décadas demonstrou a existência de, no mínimo, dois padrões: um, de aumentos súbitos e marcados, bem documentados no Reino Unido, Austrália e Nova Zelândia nos anos 60 e, novamente, na década seguinte na Nova Zelândia, e outro, de aumento gradual, detectado em inúmeros países ${ }^{(1)}$.

Mais recentemente, progressivo declínio das taxas de mortalidade tem sido observado em muitos países, como Austrália, Canadá, Alemanha, Inglaterra e Gales(2), porém em outros ainda persiste a tendência de aumento. Nos Estados Unidos, em face do aumento da prevalência, da mortalidade e número de consultas por asma, podese dizer que o problema aumentou em cerca de $50 \%$ na última década, com tendência à estabilização desde $1988^{(2)}$. O coeficiente de morte por asma em menores de 34 anos de idade, por grupos de 100.000 , subiu de 0,26 em 1980 para 0,49 em 1991 e para 0,55 em 1994, configurando uma estabilização menos evidente na faixa etária de cinco a 34 anos $^{(2)}$, grupo em que os dados dos certificados de óbitos por asma são mais confiáveis ${ }^{(3-5)}$.

Na América Latina, os dados são escassos e os trabalhos disponiveis nem sempre representam a totalidade das informações nacionais. Levantamento incluindo 12 países detectou que o número médio de mortes por asma é menor nos países do Cone Sul (Argentina, Uruguai, Paraguai e Chile) do que o das demais regiões (Colômbia, Peru e Venezuela) e da América Central (Cuba e Costa Rica) e México. A média geral do continente foi de 3,14, semelhante à do Reino Unido, mais baixa que a da Austrália, Nova Zelândia e Japão, mas mais alta que a dos Estados Unidos e Canadá(6).

Os dados sobre os óbitos no Brasil, divulgados pelo Ministério da Saúde, são de abrangência apenas parcial, pois o Sistema Nacional de Informações de Mortalidade não tem cobertura integral em vários Estados do Norte e Nordeste $e^{(7)}$. Essa não é a situação encontrada no Rio Grande do Sul e em alguns outros Estados do país, onde a abrangência do Sistema é total e o percentual de subregistro de mortes é considerado pequeno(8).

Desse modo, com alguns cuidados metodológicos é possivel estudar a morte por asma no Rio Grande do Sul. Já existem informações esparsas sobre vários aspectos da morbidade e de prevalência ${ }^{(9-13)}$. O presente trabalho atualiza para 1998 os estudos prévios referentes à mortalidade por essa doença no Estado(14).

\section{MATERIAL E MÉTODOS}

Foram estudados todos os óbitos por asma ocorridos no Estado do Rio Grande do Sul, entre 1970 e 1998, na população de cinco a 19 anos de idade.

Para tanto, foram revisados os registros de óbitos arquivados na Divisão de Informações em Saúde (DIS) da
Siglas e abreviaturas utilizadas neste trabalho

MAPE - Percentual de erro médio absoluto

MAD - Desvio médio absoluto

DIS - Divisão de Informações em Saúde

SSMA-RS - Secretaria da Saúde e Meio Ambiente do Estado do Rio Grande do Sul

MSD - Desvio mínimo quadrático

Secretaria da Saúde e Meio Ambiente do Estado do Rio Grande do Sul (SSMA-RS), identificando-se as mortes que tinham asma (para o período $1970-79$ : $\mathrm{CID}_{8}$; para 197995: $\mathrm{CID}_{9}$ 493; para 1996-98: $\mathrm{CID}_{10}$ J45) como causa básica, ocorridas em todo o Estado do Rio Grande do Sul(8). Trabalhou-se com a faixa etária de cinco a 19 anos para comparação com trabalhos anteriores realizados pelo mesmo grupo de investigadores ${ }^{(14)}$.

Foi escolhido o período de 1970 (ano de início da publicação regular das Estatísticas de Saúde do Estado do Rio Grande do Sul e da adoção do formulário padrão para Declaração de Óbito) até 1998 (último ano de publicação oficial das informações de mortalidade no Estado).

Calcularam-se os coeficientes de mortalidade dos casos de asma, utilizando os dados dos Recenseamentos Gerais do Brasil de 1970, 1980 e 1991 e das projeções populacionais para os anos intercensitários. Para avaliar o processo evolutivo da taxa de mortalidade por asma no período considerado, estudaram-se as componentes tendência e aleatória de uma série temporal (15).

A técnica de ajuste da componente tendência utilizada no presente estudo foi a dos mínimos quadrados, que foi aplicada às funções linear, exponencial, logística e quadrática. O ano de 1970 foi considerado inicial e o de 1998 o ano $n^{\circ} 29$. O estudo comparativo dos modelos ajustados foi realizado através do coeficiente de determinação $\left(\mathrm{r}^{2}\right)$ e pelas várias definições do erro residual (relativo, absoluto e quadrático).

Com a finalidade de provocar amortecimento na variabilidade da série, foi aplicado o método de médias móveis de tamanho três. Com isso, obteve-se melhor aderência dos novos dados nas equações de tendências, com a redução da componente aleatória.

Em todas as etapas, foi utilizado o software Minitab for Windows versão 12.1 .

\section{REsUlTADOS}

Ocorreram 157 mortes por asma dentre 53.221 óbitos no grupo de cinco a 19 anos, significando mortalidade proporcional de 0,29\% e variando anualmente de 0,06 a $0,64 \%$. O número médio anual de mortes por asma foi de $5,4 \pm 2,8$. Os coeficientes variaram entre $0,04 e$ $0,399 / 100.000$. 
Apresentam-se na Tabela 1 os dados referentes às freqüências absolutas, populações e coeficientes dos óbitos por asma na faixa etária estudada.

As equações de tendências com seus respectivos erros residuais e valores do coeficiente de determinação estão apresentados na Tabela 2 .

Dos ajustes realizados, as melhores medidas representativas foram para os modelos logístico e quadrático. Os valores de maior acurácia ficaram para o modelo logístico com coeficiente de determinação $\left(\mathrm{r}^{2}\right)=0,593$, percentual de erro médio absoluto (MAPE) $=23,48$, desvio médio absoluto $(\mathrm{MAD})=0,035$ e desvio mínimo quadrático $(\mathrm{MSD})=0,0021$, enquanto para o quadrático com $\mathrm{r}^{2}=$ 0,593, MAPE $=26,00, \mathrm{MAD}=0,034$ e MSD $=0,002$.

As tendências que puderam ser aceitas pelo critério de boa representatividade mostraram significativo aumento nos coeficientes de morte por asma no final da década de 70 e posterior estabilização, porém em patamar nitidamente superior, a partir do início da década de 90. As

\section{TABELA 1}

Óbitos por asma entre cinco e 19 anos de idade Rio Grande do Sul - 1970-1998

\begin{tabular}{|c|c|c|}
\hline Ano & $\begin{array}{l}\text { Número de } \\
\text { mortes }\end{array}$ & $\begin{array}{l}\text { Coeficiente de } \\
\text { mortalidade* }\end{array}$ \\
\hline 1970 & 1 & 0,040 \\
\hline 1971 & 2 & 0,080 \\
\hline 1972 & 2 & 0,080 \\
\hline 1973 & 10 & 0,399 \\
\hline 1974 & 3 & 0,119 \\
\hline 1975 & 1 & 0,040 \\
\hline 1976 & 2 & 0,079 \\
\hline 1977 & 1 & 0,040 \\
\hline 1978 & 4 & 0,158 \\
\hline 1979 & 6 & 0,236 \\
\hline 1980 & 5 & 0,196 \\
\hline 1981 & 5 & 0,196 \\
\hline 1982 & 4 & 0,156 \\
\hline 1983 & 7 & 0,273 \\
\hline 1984 & 9 & 0,350 \\
\hline 1985 & 5 & 0,194 \\
\hline 1986 & 8 & 0,310 \\
\hline 1987 & 5 & 0,193 \\
\hline 1988 & 4 & 0,154 \\
\hline 1989 & 11 & 0,384 \\
\hline 1990 & 4 & 0,137 \\
\hline 1991 & 9 & 0,339 \\
\hline 1992 & 9 & 0,338 \\
\hline 1993 & 6 & 0,222 \\
\hline 1994 & 11 & 0,293 \\
\hline 1995 & 7 & 0,254 \\
\hline 1996 & 6 & 0,220 \\
\hline 1997 & 4 & 0,290 \\
\hline 1998 & 6 & 0,179 \\
\hline
\end{tabular}

* por 100.000
Figuras 1, 2 e 3 mostram as curvas de tendência para os três modelos teóricos ajustados com os dados amortecidos que apresentaram melhores medidas de aderência.

Os resultados apontados pelo modelo logístico mostraram que provavelmente um platô esteja sendo atingido no que se refere aos coeficientes de morte por asma ano grupo de cinco a 19 anos de idade. Como o modelo quadrático também mostrou valores aceitáveis de acurácia, uma possibilidade alternativa é a de que inclusive um decréscimo esteja se iniciando. Neste modelo, o ponto de máximo global teórico ficou próximo ao $25^{\circ}$ ano (1994).

\section{DISCUSSÃO}

Apesar de reconhecidas limitações, o atestado de óbito por asma tem servido como importante instrumento para a quantificação do problema asma nas comunidades ${ }^{(5)}$.

Existem estudos mostrando que, na faixa etária de cinco a 34 anos, é um documento de acurácia satisfatória e que provavelmente não introduz artefatos significativos. As várias modificações no Código Internacional de Doenças ocorridas nos últimos 30 anos $\left(\mathrm{CID}_{8}\right.$ a $\left.\mathrm{CID}_{10}\right)$ podem ter introduzido algum aumento no registro do número de mortes por asma, mas não na faixa etária aqui considera$\mathrm{da}^{(3-5,16-18)}$.

O impacto de eventual desvio de hábito de notificação por parte dos médicos também é considerado pouco importante, uma vez que o estudo das tendências de registro de morte por bronquite e por pneumonia e a comparação com as curvas dos coeficientes de morte por asma indicam que essa também não parece ser uma explicação satisfatória.

Aumento na prevalência da doença, da gravidade, mudança de critérios de internação e terapêuticos são outras

TABELA 2

Óbitos por asma entre cinco e 19 anos de idade Rio Grande do Sul - 1970-1998

Erros residuais e coeficientes de determinação

\begin{tabular}{lrrrr}
\hline Modelo & MAPE & MAD & MSD & $\mathbf{r}^{\mathbf{2}}$ \\
\hline Logística & 23,48 & 0,035 & 0,002 & 0,593 \\
Quadrática & 26,00 & 0,034 & 0,002 & 0,593 \\
Exponencial & 28,64 & 0,044 & 0,003 & 0,490 \\
& \multicolumn{3}{c}{ Equações } \\
\hline
\end{tabular}

Logística $\quad Y_{t}=\left(10^{0}\right) /\left[3,78651+16,6591\left(0,772303^{t-1}\right)\right]$

Quadrática $\quad Y_{t}=0,0807+0,0145 t-0,000286 t^{2}$

Exponencial $\quad Y_{t}=0,111316\left(1,04042^{t}\right)$

$r^{2}=$ Coeficiente de determinação

MAPE $=$ percentual de erro médio absoluto

$\mathrm{MAD}=$ desvio médio absoluto

$\mathrm{MSD}=$ desvio mínimo quadrático 


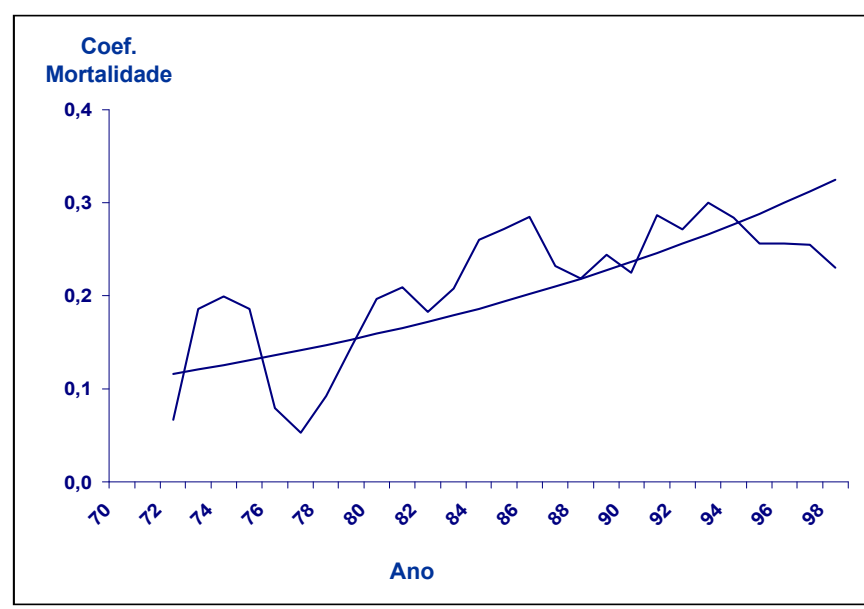

Figura 1 - Tendência da mortalidade por Asma. Modelo exponencial.

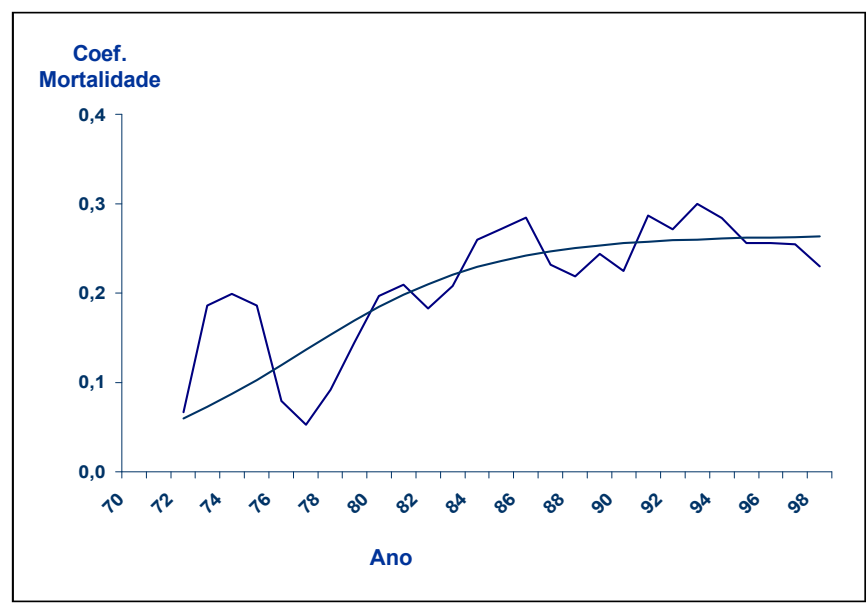

Figura 3 - Tendência da Mortalidade por Asma. Modelo logístico.

possibilidades teóricas que ainda requerem melhor avaliação como possíveis explanações para os achados.

Não se detectaram vieses que pudessem ter interferido no registro do incremento das taxas de morte por asma entre crianças e adultos jovens no Rio Grande do Sul, descrito anteriormente ${ }^{(14)}$, tampouco na aparente estabilização dos índices agora demonstrada. Ainda que não tenha sido especificamente testada, a acurácia de tais documentos no Rio Grande do Sul é considerada boa neste Estado ${ }^{(19)}$. Além disso, o incremento do número de mortes ocorreu antes mesmo de 1979 , ano da mudança do $\mathrm{CID}_{8}$ para $\mathrm{CID}_{9}$. A utilização do $\mathrm{CID}_{10}$ não parece ter introduzido nenhum problema para a codificação da asma. Também desvio de codificação de outros diagnósticos, como pneumonia e bronquite, principalmente em crianças menores, para o de asma parece não ter ocorrido, uma vez que o número de óbitos por essas patologias encontra-se estável ao longo do período estudado ${ }^{(14,20)}$.

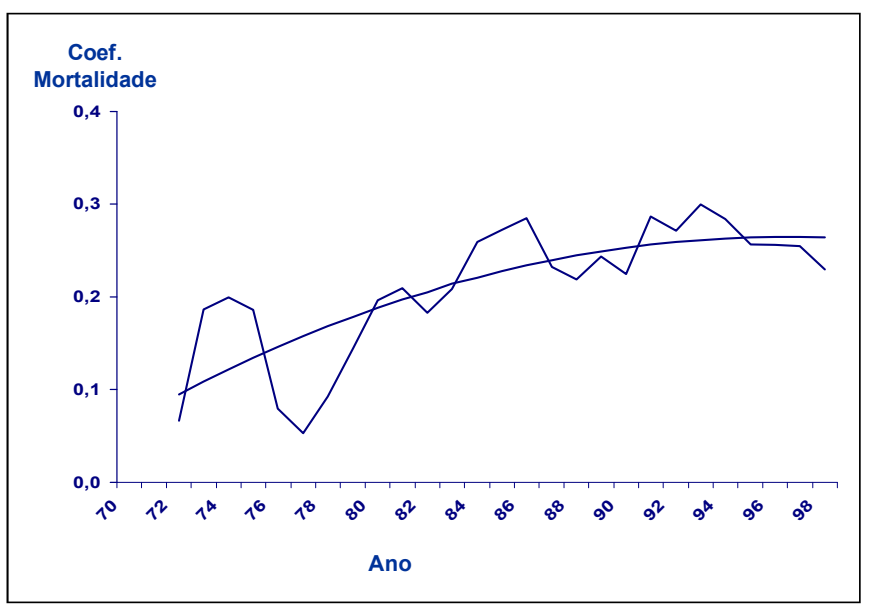

Figura 2 - Tendência da Mortalidade por Asma. Modelo quadrátiCO.

Mudanças na prevalência, morbidade e gravidade da doença têm sido consideradas como também possiveis causas do aumento da mortalidade. Nesta região do Brasil, a prevalência de asma ativa em escolares subiu de $10,9 \%$ para $22 \%$ no intervalo $1989-1998^{(12)}$, aumentando assim teoricamente o número de crianças sob risco.

Os dados encontrados neste trabalho confirmam o nítido aumento da mortalidade por asma em crianças e adolescentes no Rio Grande do Sul entre 1970 e 1992, cerca de $2 \%$ ao ano, evoluindo para relativa estabilidade a partir da segunda metade da década de 90, mas em patamar bastante mais alto que o platô anterior.

São coeficientes mais altos que os registrados no Estado de São Paulo $(0,27 / 100.000)^{(21)}$ e no Paraná $(0,24 /$ $100.000)^{(22)}$, mas todos eles podem ainda ser classificados como de baixa, porém não desprezivel magnitude. Entretanto, nesses dois Estados, os coeficientes encontram-se estáveis há vários anos, situação que só a partir da segunda metade da década de 90 parece estar ocorrendo no Rio Grande do Sul.

Os modelos teóricos aqui utilizados pela sua representatividade na aderência aos dados permitem supor que, a partir de 1994, atingiu-se uma provável estabilização da taxa de mortalidade por asma na faixa etária considerada, possivelmente em torno de 0,26/100.000, ou talvez um leve declínio do problema. Essa conclusão fica evidente pela duas melhores funções ajustadas (logística e quadrática) ao comportamento real, quando se obteve respectivamente uma assíntota de 0,26 e um ponto máximo global do mesmo valor.

Avaliações periódicas serão necessárias para o esclarecimento da real tendência evolutiva do fenômeno. É possível que os novos recursos terapêuticos, com sua utilização padronizada pelas equipes de saúde através dos vários consensos estaduais e nacionais, e a multiplicação 
dos programas educacionais comunitários já estejam interferindo na mortalidade por asma, mesmo que esse não seja o objetivo principal de tais consensos.

\section{REFERÊNCIAS}

1. Sears MR. Epidemiological trends in asthma. Can Respir J 1996;3: 261-266.

2. Sly RM, O'Donnell R. Stabilization of asthma mortality. Ann Allergy Asthma Immunol 1997;78:347-354.

3. British Thoracic Society. Accuracy of death certificates in bronchial asthma. Thorax 1984;39:505-509.

4. Barger LW, Vollmer WM, Felt RW, Buist AS. Further investigation into the recent increase in asthma death rates: a review of 41 asthma deaths in Oregon in 1982. Ann Allergy 1988;60:31-39.

5. Vollmer WM, Osborne ML, Buist AS. Uses and limitations of mortality and health care utilization statistics in asthma research. Am J Respir Crit Care Med 1994;149:579-587.

6. Neffen HE, Baena-Cagnani CE. Asthma mortality in Latin America. In: Neffen HE, Baena-Cagnani CE, Fabbri L, Holgate S, O'Byrne P, eds. Asthma - A link between environment, immunology and the airways. Seattle: Hogrefe \& Huber Publishers, 1999;231-233.

7. Ministério da Saúde. Secretaria Nacional de Ações Básicas de Saúde. Estatísticas de Mortalidade. Brasília: Centro de Documentação do Ministério da Saúde, 1985-1988;4 v.

8. Secretaria da Saúde e do Meio Ambiente do Estado do Rio Grande do Sul. Estatísticas de Saúde - Mortalidade. Porto Alegre: CORAG, 1970$1992 ; 23 \mathrm{v}$.

9. Chatkin JM, Orlandini l, Scliar MJ. A inclusão da asma brônquica nos programas de controle de doenças respiratórias agudas. J Pneumol 1986; 12:167-169.
10. Chatkin JM, Zaslavsky C, Scliar MJ. Doenças respiratórias agudas no Rio Grande do Sul, Brasil. Bol Oficina Sanit Panam 1987;102:340344.

11. Fritscher CC, Severo RD, Fagondes S, et al. Modificações na prevalência de asma brônquica em escolares de Porto Alegre. J Pneumol 1994;20:6-10

12. Fiore RM. Modificações na prevalência de asma e atopia em amostra de escolares de Porto Alegre. (Mestrado em Pediatria). Pontifícia Universidade Católica do Rio Grande do Sul, 1999.

13. Santana JCB, Menna Barreto S, Carvalho PRA. Fatores associados com asma aguda grave na infância - Aspectos epidemiológicos e clínicos. J Pediatr 1997;73:324-334.

14. Chatkin JM, Menna Barreto S, Fonseca NA, Gutierrez CA, Sears MR Trends in asthma mortality in young people in southern Brazil. Ann Allergy Asthma Immunol 1999;82:287-292.

15. Altman DG. Practical statistics for medical research. New York: Chapman and Hall, 1991.

16. Hunt Jr LW, Silverstein MD, Reed CE, et al. Accuracy of the death certificate in a population-based study of asthmatic patients. JAMA 1993;269:1947-1952.

17. Guite HF, Burney PG. Accuracy of recording of deaths from asthma in the UK: the false negative rate. Thorax 1996;51:924-928.

18. Sears M, Rea H, Boer G, et al. Accuracy of certification of deaths due to asthma. A national study. Am J Epidemiol 1986;124:1004-1011.

19. Molinari JF, Chatkin JM. Tendência da mortalidade por asma no Rio Grande do Sul. J Pneumol 1995;21:103-106.

20. Chatkin JM. Letter to the Editor. Ann Allergy Asthma Immunol 1999; 83:573.

21. Naspitz C, Solé D, Salto Jr J. Asthma mortality trends and beta 2 agonists sales in São Paulo, Brazil. J Allergy Clin Immunol 1994;94: 677.

22. Zulato S, Carvalho D, Ribeiro AC, Rosário F $^{\circ}$ NA. Morbi-mortalidade por asma no Estado do Paraná e município de Curitiba no período 1984 e 1995. J Pneumol 1999;25:12-16. 